\title{
Solving coupled Lane-Emden equations arising in catalytic diffusion reactions by reproducing kernel Hilbert space method
}

\author{
Tianchu $\mathrm{HaO}^{1, \mathrm{a}}$ and Songhe Song ${ }^{1, \mathrm{~b}}$ \\ ${ }^{1}$ College of Science, National University of Defense Technology, Changsha 410073, PR China \\ amrigant@sina.com, bshsong@nudt.edu.cn
}

Keywords: coupled Lane-Emden equation, boundary value problem, singular behavior, reproducing kernel Hilbert space method

\begin{abstract}
In this paper, we solve coupled Lane-Emden equations arising in catalytic diffusion reaction by reproducing kernel Hilbert space method. First, we construct a reproducing kernel Hilbert space satisfying boundary value conditions and provide an iterative method to obtain the approximate solution. Then we present four numerical example to show our method is accurate and reliable.Our method overcomes the singular behavior at $\mathrm{x}=0$. Finally we compare our method with ADM and Modified ADM on the maximal error remainder, which demonstrates an approximate exponential rate of convergence.
\end{abstract}

\section{Introduction}

The main purpose of this work is to solve coupled Land-Emden equations given in the form

$$
\begin{aligned}
& u^{\prime \prime}(x)+\frac{k_{1}}{x} u^{\prime}(x)=f_{1}(u, v), 0<x \leq 1, \\
& v^{\prime \prime}(x)+\frac{k_{1}}{x} v^{\prime}(x)=f_{2}(u, v), 0<x \leq 1,
\end{aligned}
$$

Subject to the initial conditions

$$
\begin{aligned}
& u^{\prime}(0)=0, u(1)=\alpha_{1}, \\
& v^{\prime}(0)=0, v(1)=\alpha_{2} .
\end{aligned}
$$

Where $k_{1}$ and $k_{2}$ are constants. $f_{1}(u, v)$ and $f_{2}(u, v)$ are analytic functions in $u$ and $v$. Such boundary value problems arise in catalytic diffusion reaction [1,2].

The Lane-Emden equation was first studied by Jonathan Homer Lane and Robert Emden [3, 4], where they considered the thermal behavior of a spherical cloud of gas acting under the mutual attraction of its molecules and subject to the classical laws of thermodynamics [5]. It also has been applied to many other fields such as mathematical physics and astrophysics $[6,7,8,9]$. Systems of Lane-Emden equations which arise in physics [1] have been studied by Adomian decomposition method(ADM) [2, 9]. The main difficulty to solve Lane-Emden equations is singular behavior at $x=0$.

Applying reproducing kernel theorem, we construct a function space named reproducing kernel space in which all function satisfy Eq.(2), then we can solve Eq.(1) in the function space, so the method is called reproducing kernel Hilbert space method. In Section 2, we construct a reproducing kernel Hilbert space and provide a numerical method for solving coupled Lane-Emeden equations with initial boundary value conditions. In Section 3, we apply the method to solve four coupled Lane-Emden equations and compare the approximate solutions with the exact solutions. In the rest part, we compare our method with ADM and Modified ADM [2] on the maximal error remainder 
parameters, which demonstrate an approximate exponential rate of convergence. In Section 4, we come to a brief conclusion.

\section{The method for solving coupled Lane-Emden equations}

Perform the following transformation to Eq.(1)

$$
\begin{aligned}
& x u^{\prime \prime}(x)+k_{1} u^{\prime}(x)=x f_{1}(u, v), 0 \leq x \leq 1, \\
& x v^{\prime \prime}(x)+k_{2} v^{\prime}(x)=x f_{2}(u, v), 0 \leq x \leq 1 .
\end{aligned}
$$

With the initial conditions

$$
\begin{aligned}
& u^{\prime}(0)=0, u(1)=\alpha_{1}, \\
& v^{\prime}(0)=0, v(1)=\alpha_{2} .
\end{aligned}
$$

Obviously the solution of Eq.(3) is the solution of Eq.(1). Let $p(x)=u(x)-\alpha_{1}$ and $q(x)=v(x)-\alpha_{2}$, then we can obtain

$$
\begin{aligned}
& x p^{\prime \prime}(x)+k_{1} p^{\prime}(x)=g_{1}(p, q, x), 0 \leq x \leq 1, \\
& x q^{\prime \prime}(x)+k_{2} q^{\prime}(x)=g_{2}(p, q, x), 0 \leq x \leq 1 .
\end{aligned}
$$

With the boundary conditions

$$
\begin{aligned}
& p^{\prime}(0)=0, p(1)=0 \\
& q^{\prime}(0)=0, q(1)=0
\end{aligned}
$$

Where $g_{1}(p, q, x)=x f_{1}\left(p+a_{1}, q+\alpha_{2}\right)$ and $g_{2}(p, q, x)=x f_{2}\left(p+\alpha_{1}, q+\alpha_{2}\right)$. To solve Eq.(1), we just need to solve Eq.(5). In the rest of this part we study the method for solving Eq.(1) with the boundary conditions Eq.(6).

The construction of the reproducing kernel Hilbert space satisfying boundary conditions. We construct a reproducing kernel Hilbert space in which the functions satisfy the boundary conditions. The function space $H^{3}[0,1]$ is defined by

$$
H^{3}[0,1]=\left\{p(x) \mid p^{\prime \prime}(x) \text { is absolutely continuous on }[0,1], p^{(3)}(x) \in L^{2}[0,1], p^{\prime}(0)=0, p(1)=0\right\}
$$

The inner product and norm in $H^{3}[0,1]$ are defined by

$$
\begin{aligned}
& <p(x), q(x)>_{H^{3}}=p(0) q(0)+p^{\prime \prime}(0) q^{\prime \prime}(0)+\int_{0}^{1} p^{(3)}(x) q^{(3)}(x) d x \\
& \|p(x)\|_{H^{3}}=\sqrt{<p(x), p(x)>_{H^{3}}}
\end{aligned}
$$

Theorem $1 H^{3}[0,1]$ is a reproducing kernel Hilbert space. That is, there exists a function $R_{x}(y) \in H^{3}[0,1]$, for any $p(y) \in H^{3}[0,1]$, such that $\left\langle p(y), R_{x}(y)>_{H^{3}}=p(x) . R_{x}(y)\right.$ can be presented by 


$$
R_{x}(y)=\left\{\begin{array}{l}
h(x, y), x<y, \\
h(y, x), x \geq y .
\end{array}\right.
$$

Where $h(x, y)=-\frac{1}{18720}(y-1)\left(\left(30 x^{2}+10 x^{3}-5 x^{4}\right)\left(-120-120 y+6 y^{2}-4 y^{3}+y^{4}\right)+\left(120+x^{5}\right)(36+\right.$ $\left.\left.36 y+6 y^{2}-4 y^{3}+y^{4}\right)\right)$.

For the proof of this theorem and the method of obtaining reproducing kernel $R_{x}(y)$, refer to $[10,11]$.

The function space $H^{1}[0,1]$ is defined by

$$
H^{1}[0,1]=\left\{p(x) \mid p(x) \text { is absolutely continuous on }[0,1], p^{\prime}(x) \in L^{2}[0,1]\right\}
$$

The inner product and norm in $H^{1}[0,1]$ are defined as follows

$$
\begin{aligned}
& <p(x), q(x)>_{H^{1}}=p(0) q(0)+\int_{0}^{1} p^{\prime}(x) q^{\prime}(x) d x \\
& \|p(x)\|_{H^{1}}=\sqrt{<p(x), p(x)>_{H^{1}}}
\end{aligned}
$$

Similarly we can prove that $H^{1}[0,1]$ is a reproducing kernel Hilbert space and its reproducing kernel can be expressed by

$$
r_{x}(y)=\left\{\begin{array}{l}
1+x, x<y \\
1+y, x \geq y
\end{array}\right.
$$

Numerical method for solving Eq.(5) . Operator $L$ is defined as follows

$$
L p(x) \triangleq x p "(x)+k_{1} p^{\prime}(x)
$$

It is clear that $L: H^{3}[0,1] \rightarrow H^{1}[0,1]$ is a linear bounded operator.

Lemma 1 Assume that $\left\{x_{i}\right\}_{i=1}^{\infty}$ is a countable dense subset in $[0,1], L^{*}$ is the adjoint operator of $L$

$$
\psi_{i}=\left(L R_{x}\right)\left(x_{i}\right) \quad i=1,2, \ldots
$$

Then $\left\{\psi_{i}\right\}_{i=1}^{\infty}$ is complete system in $H^{3}[0,1]$.

Proof. $\forall p \in H^{3}[0,1]$, if $\left\langle p, \psi_{i}\right\rangle_{H^{3}}=0$, then

$$
0=<p, \psi_{i}>_{H^{3}}=<p, L^{*} r_{x_{i}}>_{H^{3}}=<L p, r_{x_{i}}>_{H^{1}}=(L p)\left(x_{i}\right), i=1,2, \ldots
$$

Notice that $\left\{x_{i}\right\}_{i=1}^{\infty}$ is a countable dense subset in $[0,1]$, hence $L p(x) \equiv 0$, it follows that $p(x) \equiv 0$ due to the existence of $L^{-1}$.

Applying Gram-Schmidt process, we can obtain an orthogonal basis $\left\{\tilde{\psi}_{i}\right\}_{i=1}^{\infty}$ in $H^{3}[0,1]$, and

$$
\tilde{\psi}_{i}=\sum_{k=1}^{i} \beta_{i k} \psi_{k}, i=1,2, \ldots
$$


Where $\left\{\beta_{i k}\right\}$ are orthogonal coefficients.

Theorem 2 The solution of the first equation of Eq.(5) can be expressed by

$$
p(x)=\sum_{i=1}^{\infty} \sum_{k=1}^{i} \beta_{i k} g_{1}\left(p\left(x_{k}\right), q\left(x_{k}\right), x_{k}\right) \tilde{\psi}_{i} \text {. }
$$

\section{Proof.}

$$
\begin{aligned}
& p(x)=\sum_{i=1}^{\infty}<p, \tilde{\psi}_{i}>_{H^{3}} \tilde{\psi}_{i}=\sum_{i=1}^{\infty}<p, \sum_{k=1}^{i} \beta_{i k} \psi_{k}>_{H^{3}} \tilde{\psi}_{i}=\sum_{i=1}^{\infty} \sum_{k=1}^{i} \beta_{i k}<p, \psi_{k}>_{H^{3}} \tilde{\psi}_{i} \\
& =\sum_{i=1}^{\infty} \sum_{k=1}^{i} \beta_{i k}<p, L^{*} r_{x_{k}}>_{H^{3}} \tilde{\psi}_{i}=\sum_{i=1}^{\infty} \sum_{k=1}^{i} \beta_{i k}<L p, r_{x_{k}}>_{H^{1}} \tilde{\psi}_{i}=\sum_{i=1}^{\infty} \sum_{k=1}^{i} \beta_{i k}\left((L p)\left(x_{k}\right)\right) \tilde{\psi}_{i} \\
& =\sum_{i=1}^{\infty} \sum_{k=1}^{i} \beta_{i k} g_{1}\left(p\left(x_{k}\right), q\left(x_{k}\right), x_{k}\right) \tilde{\psi}_{i} .
\end{aligned}
$$

Similarly we can obtain the solution of the second equation of Eq.(5)

$$
q(x)=\sum_{i=1}^{\infty} \sum_{k=1}^{i} \gamma_{i k} g_{2}\left(p\left(x_{k}\right), q\left(x_{k}\right), x_{k}\right) \tilde{\varphi}_{i}
$$

Where $\left\{\gamma_{i k}\right\}$ are orthogonal coefficients and $\left\{\tilde{\varphi}_{i}\right\}_{i=1}^{\infty}$ is a corresponding orthogonal basis.

By truncating Fourier series Eq.(14) and Eq.(15) we can obtain the approximate solution of Eq.(5)

$$
\begin{aligned}
& p(x)=\sum_{i=1}^{n} \sum_{k=1}^{i} \beta_{i k} g_{1}\left(p\left(x_{k}\right), q\left(x_{k}\right), x_{k}\right) \tilde{\psi}_{i}, \\
& q(x)=\sum_{i=1}^{n} \sum_{k=1}^{i} \gamma_{i k} g_{2}\left(p\left(x_{k}\right), q\left(x_{k}\right), x_{k}\right) \tilde{\varphi}_{i} .
\end{aligned}
$$

Which shows that $\left\|p_{n}(x)-p(x)\right\|_{H^{3}} \rightarrow 0$ and $\left\|q_{n}(x)-q(x)\right\|_{H^{3}} \rightarrow 0$ in $H^{3}[0,1]$ as $n \rightarrow \infty$. We come to the next conclusion.

Theorem 3 The approximate solution $p_{n}(x)$ uniformly converges to exact solution $p(x)$.

Proof. Noting that

$$
\left|p_{n}(x)-p(x)\right|=\left|<p_{n}-p, R_{x}>_{H^{3}}\right| \leq\left\|p_{n}-p\right\|_{H^{3}} \cdot\left\|R_{x}(\cdot)\right\|_{H^{3}}=\sqrt{R_{x}(x)}\left\|p_{n}-p\right\|_{H^{3}}
$$

Since $R_{x}(y)$ is continuous on $[0,1]$, it follows that $\sqrt{R_{x}(x)} \leq C_{0}$, where $C_{0}$ is a constant. Combine with $\left\|p_{n}(x)-p(x)\right\|_{H^{3}} \rightarrow 0$, then we can obtain $\left|p_{n}-p\right| \rightarrow 0$ as $n \rightarrow \infty$.

In the same manner we can prove that the approximate solution $q_{n}(x)$ uniformly converges to exact solution $q(x)$. Since $p(x)$ and $q(x)$ are unknown, we provide the following iterative method 


$$
\begin{aligned}
& p_{0}^{N}(x)=0, q_{0}^{N}(x)=0, \\
& p_{n}^{N}(x)=\sum_{i=1}^{N} \sum_{k=1}^{i} \beta_{i k} g_{1}\left(p_{n-1}^{N}\left(x_{k}\right), q_{n-1}^{N}\left(x_{k}\right), x_{k}\right) \tilde{\psi}_{i}, \\
& q_{n}^{N}(x)=\sum_{i=1}^{N} \sum_{k=1}^{i} \gamma_{i k} g_{2}\left(p_{n-1}^{N}\left(x_{k}\right), q_{n-1}^{N}\left(x_{k}\right), x_{k}\right) \tilde{\varphi}_{i}, \\
& n=0,1,2,3, \ldots
\end{aligned}
$$

So far the numerical method for solving Eq.(5) is obtained.

\section{Application}

In this section, we present several examples.

\section{Example 1}

We first study the linear coupled Lane-Emden equations

$$
\begin{aligned}
& u^{\prime \prime}+\frac{3}{x} u^{\prime}=4(u+v), \\
& v^{\prime \prime}+\frac{2}{x} v^{\prime}=-3(u+v) .
\end{aligned}
$$

With the boundary conditions $u^{\prime}(0)=0, u(1)=2, v^{\prime}(0)=0, v(1)=0$. The true solution is $u(x)=1+x^{2}, v(x)=1-x^{2}$. Using our method, take $N=21, n=0,1,2, \ldots, 60$ and $N=61$, $n=0,1,2, \ldots, 30$. Let $x_{i}=\frac{i-1}{N-1}(i=1,2, \ldots, N)$. The numerical results are shown in Table 1 and Table 2.

Table 1. Numerical results for Example 1( $\mathrm{N}=21)$

\begin{tabular}{lllll}
\hline Node & $\left|u-u_{60}^{21}\right|$ & $\left|\left(u-u_{60}^{21}\right) / u\right|$ & $\left|v-v_{60}^{21}\right|$ & $\left|\left(v-v_{60}^{21}\right) / v\right|$ \\
\hline 0.2 & 8.7 E-6 & 8.4 E-6 & $4.3 \mathrm{E}-5$ & $4.5 \mathrm{E}-5$ \\
0.4 & $7.0 \mathrm{E}-6$ & $6.0 \mathrm{E}-6$ & $3.9 \mathrm{E}-5$ & $4.6 \mathrm{E}-5$ \\
0.6 & $4.6 \mathrm{E}-6$ & $3.4 \mathrm{E}-6$ & $2.9 \mathrm{E}-5$ & $4.5 \mathrm{E}-5$ \\
0.8 & $2.0 \mathrm{E}-6$ & $1.2 \mathrm{E}-6$ & $1.5 \mathrm{E}-5$ & $4.1 \mathrm{E}-5$ \\
\hline
\end{tabular}

Table 2. Numerical results for Example 1(N=61)

\begin{tabular}{lllll}
\hline Node & $\left|u-u_{30}^{61}\right|$ & $\left|\left(u-u_{30}^{61}\right) / u\right|$ & $\left|v-v_{30}^{61}\right|$ & $\left|\left(v-v_{30}^{61}\right) / v\right|$ \\
\hline 0.2 & $9.7 \mathrm{E}-7$ & $9.3 \mathrm{E}-7$ & $4.8 \mathrm{E}-6$ & $5.0 \mathrm{E}-6$ \\
0.4 & $7.8 \mathrm{E}-7$ & $6.7 \mathrm{E}-7$ & $4.3 \mathrm{E}-6$ & $5.1 \mathrm{E}-6$ \\
0.6 & $5.1 \mathrm{E}-7$ & $3.7 \mathrm{E}-7$ & $3.2 \mathrm{E}-6$ & $5.0 \mathrm{E}-6$ \\
0.8 & $2.2 \mathrm{E}-7$ & $1.4 \mathrm{E}-7$ & $1.6 \mathrm{E}-6$ & $4.5 \mathrm{E}-6$ \\
\hline
\end{tabular}

\section{Example 2}

We study the nonlinear coupled Lane-Emden equations

$$
\begin{aligned}
& u^{\prime \prime}+\frac{1}{x} u^{\prime}=v^{3}\left(u^{2}+1\right), \\
& v^{\prime \prime}+\frac{3}{x} v^{\prime}=-v^{5}\left(u^{2}+3\right) .
\end{aligned}
$$


Subject to the boundary conditions $u^{\prime}(0)=0, u(1)=\sqrt{2}, v^{\prime}(0)=0, v(1)=\sqrt{2} / 2$. The exact solution is $u(x)=\sqrt{x^{2}+1}, v(x)=1 / \sqrt{x^{2}+1}$. Take $N=61$ and $n=0,1,2, \ldots, 50$. Let $x_{i}=\frac{i-1}{N-1}(i=1,2, \ldots, N)$. The numerical results are shown in Table 3.

Table 3. Numerical results for Example 2(N=61)

\begin{tabular}{lllll}
\hline Node & $\left|u-u_{50}^{61}\right|$ & $\left|\left(u-u_{50}^{61}\right) / u\right|$ & $\left|v-v_{50}^{61}\right|$ & $\left|\left(v-v_{50}^{61}\right) / v\right|$ \\
\hline 0.1 & $8.7 \mathrm{E}-7$ & $8.7 \mathrm{E}-7$ & $6.1 \mathrm{E}-6$ & $6.2 \mathrm{E}-6$ \\
0.3 & $8.9 \mathrm{E}-7$ & $8.6 \mathrm{E}-7$ & $5.6 \mathrm{E}-6$ & $5.9 \mathrm{E}-6$ \\
0.5 & $1.1 \mathrm{E}-6$ & $9.6 \mathrm{E}-7$ & $4.9 \mathrm{E}-6$ & $5.5 \mathrm{E}-6$ \\
0.7 & $9.6 \mathrm{E}-7$ & $7.9 \mathrm{E}-7$ & $3.3 \mathrm{E}-6$ & $4.0 \mathrm{E}-6$ \\
0.9 & $3.9 \mathrm{E}-7$ & $2.9 \mathrm{E}-7$ & $1.1 \mathrm{E}-6$ & $1.5 \mathrm{E}-6$ \\
\hline
\end{tabular}

\section{Example 3}

We next study the nonlinear coupled Lane-Emden equations

$$
\begin{aligned}
& u^{\prime \prime}+\frac{8}{x} u^{\prime}=2 u(9-2 \ln v), \\
& v^{\prime \prime}+\frac{4}{x} v^{\prime}=2 v(5-2 \ln u) .
\end{aligned}
$$

Subject to the boundary conditions $u^{\prime}(0)=0, u(1)=e^{-1}, v^{\prime}(0)=0, v(1)=e$. The exact solution is $u(x)=e^{-x^{2}}, v(x)=e^{x^{2}}$. Take $N=21$ and $n=0,1,2, \ldots, 60$. The numerical results are shown in Table 4.

Table 4. Numerical results for Example 3( $\mathrm{N}=21)$

\begin{tabular}{lllll}
\hline Node & $\left|u-u_{60}^{21}\right|$ & $\left|\left(u-u_{60}^{21}\right) / u\right|$ & $\left|v-v_{60}^{21}\right|$ & $\left|\left(v-v_{60}^{21}\right) / v\right|$ \\
\hline 0.1 & $4.3 \mathrm{E}-5$ & $4.3 \mathrm{E}-5$ & $3.1 \mathrm{E}-4$ & $3.1 \mathrm{E}-4$ \\
0.3 & $3.5 \mathrm{E}-5$ & $3.8 \mathrm{E}-5$ & $3.4 \mathrm{E}-4$ & $3.1 \mathrm{E}-4$ \\
0.5 & $2.3 \mathrm{E}-5$ & $3.0 \mathrm{E}-5$ & $4.3 \mathrm{E}-4$ & $3.3 \mathrm{E}-4$ \\
0.7 & $1.1 \mathrm{E}-5$ & $1.8 \mathrm{E}-5$ & $5.5 \mathrm{E}-4$ & $3.4 \mathrm{E}-4$ \\
0.9 & $7.7 \mathrm{E}-7$ & $1.7 \mathrm{E}-6$ & $4.4 \mathrm{E}-4$ & $2.0 \mathrm{E}-4$ \\
\hline
\end{tabular}

In some cases, we can not obtain the exact solution of the equation. The error analysis of the sequence of the analytic approximate solutions can be performed by using the maximal error reminder parameters, which demonstrate an approximate rate of convergence. Let's consider the following coupled Lane-Emden equations,

$$
\begin{aligned}
& u^{\prime \prime}(x)+\frac{k_{1}}{x} u^{\prime}(x)=\sigma_{11} u^{2}+\sigma_{12} u v, 0<x \leq 1, \\
& v^{\prime \prime}(x)+\frac{k_{1}}{x} v^{\prime}(x)=\sigma_{21} u^{2}+\sigma_{22} u v, 0<x \leq 1 .
\end{aligned}
$$

Subject to the initial conditions $u^{\prime}(0)=0, u(1)=\alpha_{1}, v^{\prime}(0)=0, v(1)=\alpha_{2}$. The parameter $\sigma_{11}, \sigma_{12}, \sigma_{21}, \sigma_{22}, \alpha_{1}, \alpha_{2}$ can be specified for the actual chemical reactions. Assume that $u_{n}(x)$ and 
$v_{n}(x)$ are the approximate solutions of Eq.(18). Let $E R_{n}^{(1)}(x)=u_{n}^{\prime \prime}(x)+\frac{2}{x} u_{n}^{\prime}(x)-\sigma_{11} u_{n}^{2}-\sigma_{12} u_{n} v_{n}$, $E R_{n}^{(2)}(x)=v_{n}^{\prime \prime}(x)+\frac{2}{x} v_{n}^{\prime}(x)-\sigma_{21} u_{n}^{2}-\sigma_{22} u_{n} v_{n}$. The maximal error reminder parameters are defined as $M E R_{n}^{(1)}=\max _{0 \leq x \leq 1}\left|E R_{n}^{(1)}(x)\right|, M E R_{n}^{(2)}=\max _{0 \leq x \leq 1}\left|E R_{n}^{(2)}(x)\right|$, which are a measure of how well the sequence of solution approximations satisfy the equations. We take $\sigma_{11}=1, \sigma_{12}=2 / 5, \sigma_{21}=1 / 2, \sigma_{22}=1, \alpha_{1}=1, \alpha_{2}=2$, we calculate the maximal error remainder parameters. We compare our method with ADM and Modified ADM. The results are shown in Table 5 and Table 6.

Table 5 The maximal error parameters $\left(M E R_{n}^{(1)}\right)$

\begin{tabular}{cccc}
\hline $\mathrm{n}$ & ADM & Modified ADM & Our method(N=21) \\
\hline 2 & 0.866667 & 0.413252 & 0.268869 \\
3 & 0.442824 & 0.236267 & 0.0810987 \\
4 & 0.230943 & 0.0643409 & 0.0232325 \\
5 & 0.126713 & 0.0479165 & 0.00684451 \\
\hline \multicolumn{4}{c}{ Table 5 The maximal error parameters $\left(M E R_{n}^{(2)}\right)$} \\
\hline 2 & ADM & Modified ADM & Our method(N=21) \\
\hline 3 & 1.14667 & 0.567430 & 0.356489 \\
4 & 0.570630 & 0.309872 & 0.107057 \\
5 & 0.0293825 & 0.0851094 & 0.0306852 \\
\hline
\end{tabular}

\section{Conclusion}

In this paper, a new method is proposed for solving coupled Land-Emden equations. First we construct a reproducing kernel space, then we give an iterative method for obtaining the approximate solutions, finally we present four numerical examples including one linear system and three nonlinear systems and compare the present method with ADM and Modified ADM. The numerical result show that the present method is an accurate and reliable analytical technique for solving coupled Lane-Emden equations. Besides our method performs well at the singular point $x=0$. Moreover our method can deal with more complicated solutions.

\section{References}

[1] Flockerzi D, Sundmacher K. On coupled Lane-Emden equations arising in dusty uid models[C]Journal of Physics: Conference Series.IOP Publishing, 2011, 268(1): 012006.

[2] Rach R, Duan J S, Wazwaz A M. Solving coupled Lane-Emden boundary value problems in catalytic di_usion reactions by the Adomian decomposition method[J]. Journal of Mathematical Chemistry, 2014, 52(1): 255-267.

[3] Lane H J. On the Theoretical Temperature of the Sun, under the Hypothesis of a gaseous Mass maintaining its Volume by its internal Heat, and depending on the laws of gases as known to terrestrial Experiment[J]. American Journal of Science, 1870 (148): 57-74.

[4] Zou H. A priori estimates for a semilinear elliptic system without variational structure and their applications[J]. Mathematische Annalen,2002, 323(4): 713-735.

[5] Richardson OW. The Emission of Electricity from Hot Bodies. Longmans Green and Co.: London, UK, 1921.

[6] Fowler R H, Nordheim L. Electron emission in intense electric fields[J]. Proceedings of the 
Royal Society of London. Series A, Containing Papers of a Mathematical and Physical Character, 1928: 173-181.

[7] Wazwaz A M. A new method for solving singular initial value problems in the second-order ordinary di_erential equations[J]. Applied Mathematics and Computation, 2002, 128(1): 45-57.

[8] Wazwaz A M. A new algorithm for solving differential equations of Lane-Emden type[J]. Applied Mathematics and Computation, 2001,118(2): 287-310.

[9] Wazwaz A M, Rach R, Duan J S. A study on the systems of the Volterra integral forms of the Lane-Emden equations by the Adomian decomposition method[J]. Mathematical Methods in the Applied Sciences, 2014, 37(1): 10-19.

[10]Cui M, Lin Y. Nonlinear Numerical Analysis in Reproducing Kernel Space[M]. Nova Science Publishers, Inc., 2009.

[11]Xinjiang Z. Theorem for reproducing kernel and applications. Science Press, 2010. 